\title{
Planar Minimally Rigid Graphs and Pseudo-Triangulations
}

\author{
Ruth Haas ${ }^{1}$ David Orden ${ }^{2}$ Günter Rote ${ }^{3}$ \\ Francisco Santos ${ }^{2}$ Brigitte Servatius ${ }^{4}$ Hermann Servatius ${ }^{4}$ \\ Diane Souvaine ${ }^{5}$ Ileana Streinu ${ }^{6}$ Walter Whiteley ${ }^{7}$
}

\begin{abstract}
Pointed pseudo-triangulations are planar minimally rigid graphs embedded in the plane with pointed vertices (incident to an angle larger than $\pi$ ). In this paper we prove that the opposite statement is also true, namely that planar minimally rigid graphs always admit pointed embeddings, even under certain natural topological and combinatorial constraints. The proofs yield efficient embedding algorithms. They also provide - to the best of our knowledge - the first algorithmically effective result on graph embeddings with oriented matroid constraints other than convexity of faces.
\end{abstract}

\section{Categories and Subject Descriptors}

F.2.2 [Theory of Computation]: Analysis of Algorithms and Problem Complexity_Geometrical problems and computations

\section{General Terms}

Algorithms, Theory

${ }^{1}$ Department of Mathematics, Smith College, Northampton, MA 01063, USA.rhaas@math.smith.edu.

${ }^{2}$ Departamento de Matematicas, Estadistica y Computacion, Universidad de Cantabria, E-39005 Santander, Spain. \{ordend,santos\}@matesco.unican.es. Supported by grant BFM2001-1153, Spanish Min. of Science and Technology

${ }^{3}$ Institut für Informatik, Freie Universität Berlin, Takustraße 9, D-14195 Berlin, Germany. rote@inf.fu-berlin.de. Partly supported by the Deutsche Forschungsgemeinschaft (DFG) under grant RO 2338/2-1.

${ }^{4}$ Mathematics Department, Worcester Polytechnic Institute, Worcester, MA 01609, USA. \{bservat,hservat\}@math. wpi.edu.

${ }^{5}$ Department of Computer Science, Tufts University, Medford MA, USA.dls@cs.tufts.edu.

${ }^{6}$ Department of Computer Science, Smith College, Northhampton, MA 01063, USA. streinu@cs.smith.edu. Supported by NSF grants CCR-0105507 and CCR-0138374.

${ }^{7}$ Department of Mathematics and Statistics, York University, Toronto, Canada. whiteley@mathstat.yorku.ca. Supported by grants from NSERC (Canada) and NIH (US).

Permission to make digital or hard copies of all or part of this work for personal or classroom use is granted without fee provided that copies are not made or distributed for profit or commercial advantage and that copies bear this notice and the full citation on the first page. To copy otherwise, to republish, to post on servers or to redistribute to lists, requires prior specific permission and/or a fee.

SoCG'03, June 8-10, 2003, San Diego, California, USA.

Copyright 2003 ACM 1-58113-663-3/03/0006 ...\$5.00.

\section{Keywords}

pseudo-triangulation, rigidity, graph drawing

\section{INTRODUCTION}

In this paper we prove that all planar minimally rigid graphs (planar Laman graphs) admit embeddings as pointed pseudo-triangulations. In contrast to the traditional planar graph embeddings, where all the faces are designed to be convex, ours have interior faces which are as non-convex as possible (pseudo-triangles). We give two proofs of independent interest. They are constructive and yield efficient embedding algorithms. We extend the result to combinatorial pseudo-triangulations, which are topological (pseudosegment) embeddings with additional partial oriented matroid information, and to rigidity circuits.

Novelty. Planar graph embeddings with non-convex faces have not been systematically studied before. Our result links them to rigidity-theoretic and matroidal properties of planar graphs. We give a simple and elegant combinatorial characterization of all the graphs which can be embedded as pointed pseudo-triangulations, answering an open question posed in [38]. In addition, to the best of our knowledge, this is the first result on algorithmically efficient graph embeddings with oriented matroid constraints, holding for an interesting family of graphs. In contrast, the universality theorem for pseudo-line arrangements of Mnëv [26] implies that the general problem of embedding graphs with oriented matroid constraints is as hard as the existential theory of the reals.

Proof Techniques and Algorithmic Results. We use two proof techniques. The first one is based on a graphtheoretic inductive construction for minimally rigid graphs (Henneberg construction), which we extend to include topological information. The second uses linear algebra and relies on an extension by [8] of Tutte's technique for spring embeddings of planar graphs. Both produce efficient embedding algorithms.

Laman Graphs and Pseudo-Triangulations. Let $G=$ $(V, E)$ be a graph with $n=|V|$ vertices and $m=|E|$ edges. $G$ is a Laman graph if $m=2 n-3$ and every subset of $k \geq 2$ vertices spans at most $2 k-3$ edges. An embedding $G(P)$ of the graph $G$ on a set of points $P=\left\{p_{1}, \ldots, p_{n}\right\} \subset R^{2}$ is a mapping of the vertices $V$ to points in the Euclidian plane $i \mapsto p_{i} \in P$. The edges $i j \in E$ are mapped to straight line segments $p_{i} p_{j}$. We say that the vertex $i$ of the embedding $G(P)$ is pointed if all its incident edges lie on one side of some 
line through $p_{i}$. Equivalently, some consecutive pair of edges adjacent to $i$ (in the circular counter-clockwise order around the vertex) spans a reflex angle. An embedding $G(P)$ is plane if no pair of segments $p_{i} p_{j}$ and $p_{k} p_{l}$ corresponding to non-adjacent edges $i j, k l \in E, i, j \notin\{k, l\}$ have a point in common. A graph $G$ is planar if it has a plane embedding. A pseudo-triangle is a simple planar polygon with exactly three convex vertices. A pseudo-triangulation of a planar set of points is a plane graph whose outer face is convex and all interior faces are pseudo-triangles. In a pointed pseudotriangulation all the vertices are pointed. See Fig. 1.

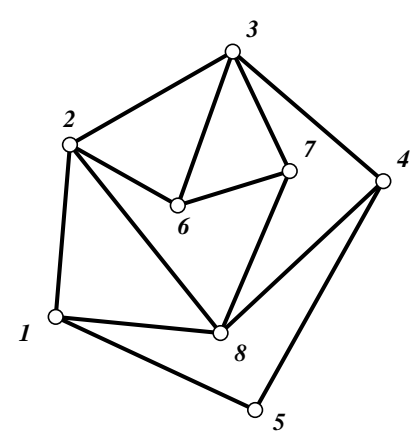

(a)

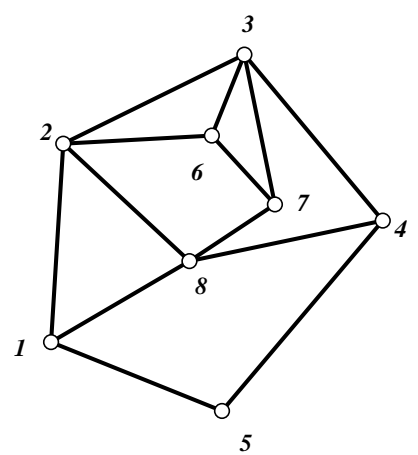

(b)
Figure 1: Two embeddings of a planar Laman graph: (a) is a pointed pseudo-triangulation, (b) is not: the faces 2876 and 1548 are not pseudo-triangles and the vertices 6 and 8 are not pointed.

Equivalent characterizations and other properties of pointed pseudo-triangulations are given in [37], where they are called minimum pseudo-triangulation. In particular, the underlying graphs of pointed pseudo-triangulations have exactly $2 n-3$ edges and are in fact Laman graphs.

Historical Perspective. Techniques from Rigidity Theory have been recently applied to problems such as collision free robot arm motion planning [9, 37], molecular conformations $[21,46,43]$ and sensor and network topologies with distance and angle constraints [11].

Laman graphs are the fundamental objects in 2-dimensional Rigidity Theory. Also known as isostatic or generically minimally rigid graphs, they characterize combinatorially the property that a graph, embedded on a generic set of points, is infinitesimally rigid (with respect to the induced edge lengths). See $[23,16,47]$. The most famous open question in Rigidity Theory (the Rigidity Conjecture, see [16]) is finding their 3-dimensional counterpart.

Pseudo-triangulations are relatively new objects, introduced and applied in Computational Geometry for problems such as visibility [30, 29, 35], kinetic data structures [3] and motion planning for robot arms [37]. They have rich combinatorial, rigidity-theoretic and polyhedral properties, many of which have only recently started to be investigated, see $[37,34,31,22,2,5,1,6]$. In particular, the fact that they are rigid graphs which become expansive mechanisms when an edge is removed from their convex hull, has proved to be crucial in designing efficient motion planning algorithms for planar robot arms, see [37]. Finding their 3-dimensional counterpart, which is perhaps the main open question about pseudo-triangulations and expansive motions, may lead to efficient motion planning algorithms for certain classes of 3dimensional linkages, with potential impact on understanding protein folding processes.

Graph Drawing is a field with a distinguished history $[12,45,44]$, and planar graph embeddings have received substantial attention in the literature $[8,15,39,10]$. Extensions of graph embeddings from straight-line to pseudoline segments have been recently considered (see e.g. [28]). It is natural to ask which such embeddings are stretchable, i.e. whether they can be realized with straight-line segments while maintaining some desired combinatorial substructure. Indeed, the primordial planar graph embedding result, Fáry's Theorem [12], is just an instance of answering such a question. Graph embedding stretchability questions have usually ignored oriented matroidal constraints, allowing for the free reorientation of triplets of points when not violating other combinatorial conditions. The notable exception concerns the still widely open visibility graph recognition problem, approached in the context of pseudo-line arrangements (oriented matroids) by [27]. In [36] it is shown that it is not always possible to realize with straight-lines a pseudo-visibility graph, while maintaining oriented matroidal constraints.

In contrast, this paper gives the first non-trivial stretchability result on a natural graph embedding problem with oriented matroid constraints. It adds to the already rich body of surprisingly simple and elegant combinatorial properties of pointed pseudo-triangulations by proving a natural connection.

Main Result. We are interested in planar Laman graphs. Not all Laman graphs fall into this category. For example, $K_{3,3}$ is Laman but not planar. But the underlying graphs of all pointed pseudo-triangulations are planar Laman. See Figure 1. We prove that the converse is always true:

ThEOREM 1.1. (Main Theorem) Every planar Laman graph can be realized as a pointed pseudo-triangulation.

We prove in fact a stronger result, allowing us to fix $a$ priori the face structure (Theorem 3.1), and even the combinatorial information regarding which vertices are convex in each face (Theorem 4.2). Finally, we answer a natural question related to the underlying matroidal structure of planar rigidity and extend the result to planar rigidity circuits, which are minimal dependent sets in the rigidity matroid where the bases (maximally independent sets) are the Laman graphs. By adding edges to a pointed (minimum) pseudo-triangulation while maintaining planarity, the graph has increased dependency level (in the rigidity matroid) and can no longer be realized with all vertices pointed, but it can always be realized with straight edges. Our concern is to maintain the minimum number of non-pointed vertices, for the given edge count. For circuits, this number is one, and we show that it can be attained.

Further Developments. Every planar graph can be embedded on a grid of size $O(n) \times O(n)$, see for example [15, $39,13]$. Here is a natural remaining problem.

Open Question 1. Can a planar Laman graph be embedded as a pseudo-triangulation on a $O\left(n^{k}\right) \times O\left(n^{k}\right)$ size grid? What is the smallest such $k$ ?

We also conjecture that the embedding algorithm can be 
improved to $O(n \log n)$ time, and present two additional algorithmic open questions in Section 3.

Organization of the paper. In section 2 we define most of the technical concepts needed later for the proof. The main result, proven with an inductive argument, is presented in section 3. Section 4 contains the more general result on combinatorial pseudo-triangulations. In section 5 we sketch the proof for the extension to planar rigidity circuits.

\section{PRELIMINARIES}

For the standard graph and rigidity-theoretic terminology used in this paper we refer the reader to [16] and [48]. For relevant facts about pointed pseudo-triangulations, see [37]. In this section we review a classical result from Rigidity Theory (Henneberg constructions) on which the approach in Section 3 is based and give new technical definitions, including the concept of purely combinatorial pseudo-triangulations. For lack of space, we do not include the technical definitions needed for Sections 4 and 5 .

Topologically Embedded Planar Graphs. Any plane embedding of a planar graph induces a topological embedding of the underlying graph $G$. This is independent of point coordinates, and captures only the combinatorial information about the faces of the embedding and their adjacencies. Equivalently, this information is captured by the rotations at each vertex: the counter-clockwise circular order of the incident edges at each vertex in the embedding. A topological embedding of a planar graph is such a system of rotations (or equivalently, the faces and their adjacencies) which can be realized in the plane with non-crossing curves (pseudo-segments). A topological embedding with a marked face contains, in addition, information about which face of the embedding is unbounded (the outer or exterior face). It is well-known that a 3-connected planar graph has a unique embedding on the sphere, but 2-connected ones (including some planar Laman graphs) may have several. The plane embedding is obtained by choosing one face as the outer face, and every topological embedding of a simple planar graph can be realized with straight-line edges in the plane, for any choice of an outer face. Traditionally, such results have focused on embeddings with convex faces, even for non-maximal planar graphs [7, 13]. In this paper we deal with a different type of embedding which is, in a sense, as non-convex as possible on the interior faces, while still non-crossing.

Faces, Angles, Corners, Pseudo-triangles and Pseudo$k$-gons. A (combinatorial) angle at a vertex $v$ incident to a face $F$ of a topologically embedded planar graph is a pair of consecutive (adjacent) edges $v u, v w$ on the face. For an embedded planar graph, an angle is convex if less than $\pi$ and reflex otherwise.

The convex vertices of a simple polygon (representing some embedded face) will be called corners. A polygon with $k$ corners is called a pseudo-k-gon. A pseudo-triangle is a polygon with three corners. The interior faces of a pseudotriangulation are pseudo-triangles. In a pointed pseudotriangulation, each vertex is adjacent to a reflex angle. The exterior face is bounded by the convex hull of the set of points. It is the only face lying outside its boundary polygon, and also the only face having no corners (as seen from inside the face).
For conciseness, in the rest of the paper we will drop pointed and refer to our objects simply as pseudo-triangulations, except in Section 5 where pseudo-triangulation will no longer mean pointed.
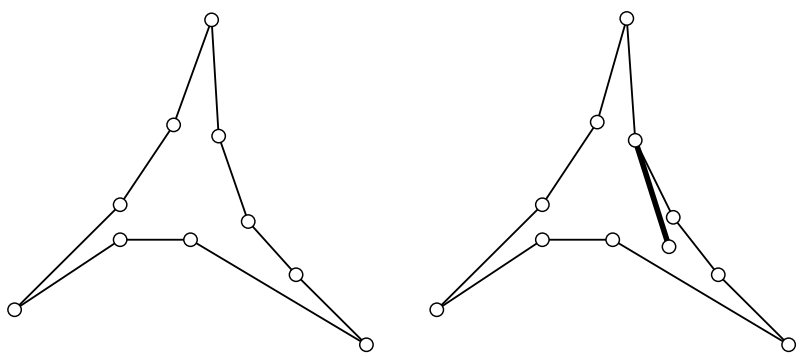

Figure 2: Left: a pseudo-triangle, with its three side chains and three corners. Right: a tangent from an interior point to one of the three side-chains.
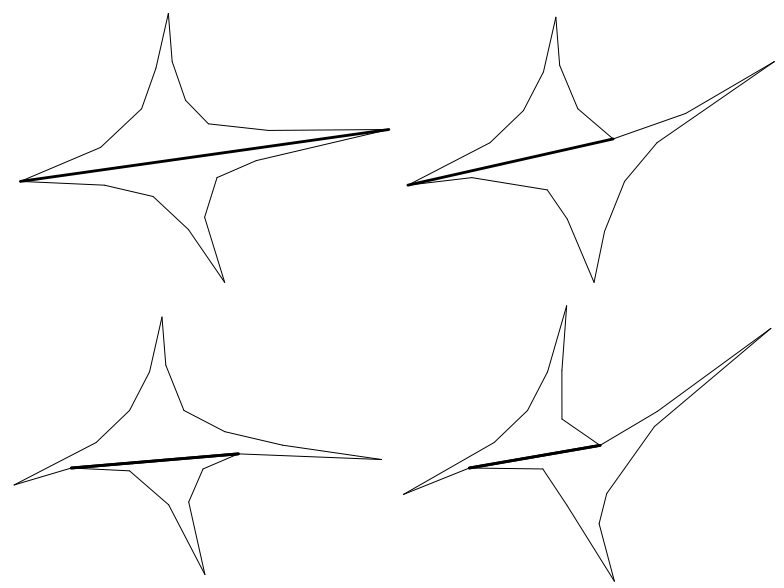

Figure 3: The four types of interior bitangents to a pseudo-4-gon.

Side chains, Tangents and Bitangents. The following concepts are needed for the proof of Lemma 3.4 of Section 3 and can be skipped at first reading.

A (side) chain of a face is the inner convex boundary between two consecutive corners. Non-corner vertices lie on exactly one chain, while corners lie on two chains, except for the unique corner of an exterior 1-gon, which lies on the unique side chain of the face (we may think of this unique chain as having multiplicity 2 with respect to the corner). For a vertex $i \in V$, denote by $p_{i}$ the corresponding point of the embedding $G(P)$ and by $C_{i}$ the chain (or chains) on which it lies. A tangent from a point $p$ interior to a face $F$ is a line segment $p p_{i}$ lying entirely inside $F$, which joins $p$ to a point $p_{i}$ on the face and whose supporting line does not cut through the chain(s) $C_{i}$ at the tangency point $p_{i}$. A bitangent to a face $F$ is a segment $p_{i} p_{j}$ lying inside $F$ and which is tangent at its endpoints $p_{i}$ and $p_{j}$ to the chains $C_{i}$ and $C_{j}$.

Notice that adding a tangent $p p_{i}$ from a new point $p$ to a pointed embedded graph maintains the pointedness of the 
vertex $p_{i}$ of tangency. See Figure 2. Adding a bitangent inside an interior face is possible only if the face is an interior $k$-gon with $k \geq 4$.

We will need the following simple classification of bitangents to a pseudo-4-gon, of which there are exactly four cases (see Figure 3): a) Both endpoints are (opposite) corners of the pseudo-4-gon. b) One endpoint is a corner and the other is not. c) Both endpoints are non-corners, and the two side chains to which they belong are consecutive and lie on the same side of the supporting line of the bitangent. d) Both endpoints are non-corners, and the two side chains to which they belong are opposite and lie on opposite sides of the supporting line of the bitangent.

Laman graphs and Henneberg constructions. Laman graphs can be characterized in a variety of ways. In particular, a Laman graph on $n$ vertices has an inductive construction as follows [18]. Start with a triangle for $n=3$. At each step, add a new vertex in one of the following two ways:

- Henneberg I (vertex addition): the new vertex is connected via two new edges to two old vertices.

- Henneberg II (edge splitting): a new vertex is added on some edge (thus splitting the edge into two new edges) and then connected to a third vertex. Equivalently, this can be seen as removing an edge, then adding a new vertex connected to its two endpoints and to some other vertex.

Our approach is inspired by the following result, stated by Henneberg [18], and by its proof, due to Tay and Whiteley [42]:

LEMma 2.1. A graph is a Laman graph if and only if it has a Henneberg construction.

See Figure 4. Part (b) shows a drawing with crossing edges, to emphasize that the Henneberg constructions hold for general, not necessarily planar Laman graphs.

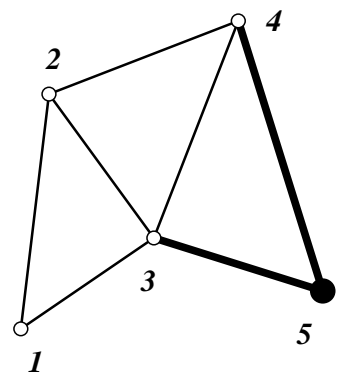

(a)

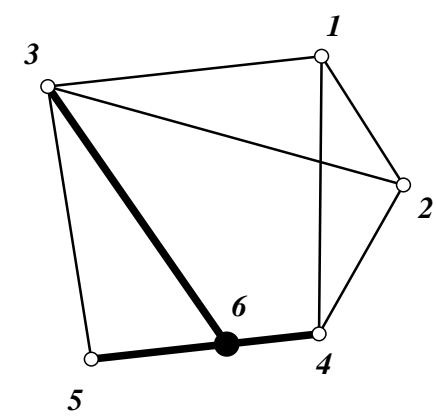

(b)
Figure 4: Illustration of the two types of steps in a Henneberg sequence, with vertices labelled in the construction order. (a) Henneberg I for vertex 5, connected to old vertices 3 and 4. (b) Henneberg II for vertex 6 , connected to old vertices 3,4 and 5 .

Combinatorial Pseudo-Triangulations. An assignment of a label $\{C, R\}$ (standing for convex and reflex) to each angle of a topologically embedded planar graph is called a combinatorial (pointed) pseudo-triangulation if:

- For each vertex, exactly one of its incident angles is labelled $R$ (pointedness).

- There exists one face (the outer face) whose angles are all labelled $R$.

- All other faces have exactly three angles labelled $C$.

A consequence of the Main Result is that every planar Laman graph has a combinatorial pseudo-triangulation assignment. In Section 4 we will mention a direct proof and we will present an algorithm for computing one, or all, of the combinatorial pseudo-triangulations compatible with a given topologically embedded planar Laman graph. In addition, in section 4 we prove that every combinatorial pseudotriangulation of a Laman graph can be realized as an actual (straight-line) pseudo-triangulation.

\section{MAIN RESULT}

In this section we give the first proof of our Main Theorem 1.1 stated in the following slightly more general form.

THEOREM 3.1. A planar Laman graph with a given topological embedding and a designated outer face can be realized as a pointed pseudo-triangulation with the same face structure.

The proof is a geometric version of the Henneberg construction, and is contained in the following three lemmas. Lemma 3.2 reduces the problem to the case of a graph with a triangular outer face. Lemma 3.3 gives a topological famework for the Henneberg construction. Lemma 3.4 performs the inductive step in the geometric realization.

Lemma 3.2. (Reduction to a Triangular Outer Face) If we know how to embed as a pseudo-triangulation a planar Laman graph with a triangular outer face, then we can solve the problem for an outer face of any size.

Proof. Suppose that an outer face with more than 3 vertices is prescribed for the graph $G_{n}$. Then we consider a Laman graph $G_{n+3}$ of $n+3$ vertices by adding 3 vertices to the outer face, connecting these three vertices into a triangle that contains the original graph, and then adding an edge from each of the new vertices to the exterior polygon of $G_{n}$. We now realize $G_{n+3}$ as a pseudo-triangulation with the new triangle as the exterior face. In this realization, the graph $G_{n}$ must be realized with its outer face convex by the following argument. The three new interior edges of $G_{n+3}$ provide two corners each in their outer end-point and at least one corner in the interior one. Since the three faces incident to them have nine corners in total, the boundary of $G_{n}$ provides no corner to the three new interior faces of $G_{n+3}$. See Figure 5 .

LEMMA 3.3. (Planar Laman graphs admit planar Henneberg constructions) Every topological planar embedding of a Laman graph with a triangular outer face has a Henneberg construction in which:

- All intermediate graphs are planarly embedded. 


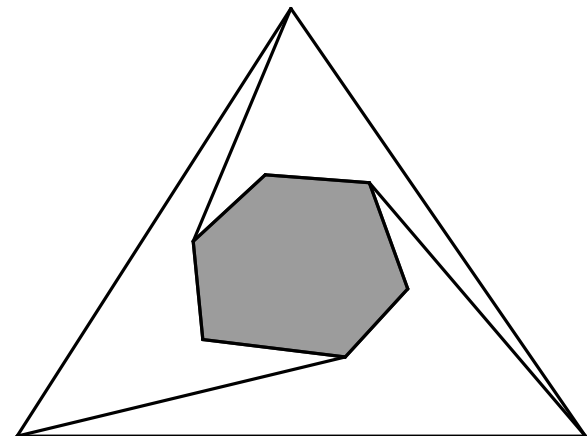

Figure 5: Reducing to a triangular outer face embedding.

- We start with the triangle corresponding to the outer face. This face will never be altered.

- At each step, a new vertex is inserted in some interior face. Only edges and faces involved in the Henneberg step are changed: either the new vertex is added inside a face of the previous graph (Henneberg I), or inside a face obtained by removing an edge between two faces of the previous graph (Henneberg II).

Proof. We work by reverse induction, along similar lines as the proof of Lemma 2.1 (see [42]). Since a Laman graph has $2 n-3$ edges, there exists a vertex $v$ of degree 2 or 3 . If it is of degree 2, removing it and its incident edges merges two faces into one, and the graph obtained is clearly Laman. If it is of degree 3 , let its neighbors be $v_{1}, v_{2}$ and $v_{3}$. Tay and Whiteley [42] prove that the removal of $v$ and the insertion of one of the three edges $v_{1} v_{2}, v_{1} v_{3}$ or $v_{2} v_{3}$, say $v_{1} v_{2}$, gives a Laman graph. Topologically, we just delete the edge $v v_{3}$ and merge its two incident faces into one. The other face incident to $v$ has two boundary edges merged into one. In either case, continue the inductive reduction until $n=3$.

This topological Henneberg construction can be performed having a given triangle as the starting graph in the Henneberg construction, and maintaining it all throughout as the outer face. Indeed, the three boundary vertices have sum of degrees at least 7 if $G$ has $n>3$ vertices. Since the total sum of degrees in the Laman graph is $4 n-6$, the $n-3$ interior vertices have degree sum at most $4 n-13=4(n-3)-1$. Hence, there is at least one interior vertex of degree at most three, at which we can perform the Henneberg deletion. This way, none of the topological steps will affect the exterior face.

LEMMA 3.4. (Inductive Extension) Let $G_{n}$ be a topologically embedded planar Laman graph obtained from $G_{n-1}$ via a Henneberg step on an interior face, and assume inductively that $G_{n-1}$ has a pseudo-triangulation embedding $G_{n-1}\left(P_{n-1}\right)$. Then there is a pseudo-triangulation embedding $G_{n}\left(P_{n}\right)$ of $G_{n}$, where $P_{n}=P_{n-1} \cup\left\{p_{n}\right\}$ is obtained by adding a new vertex $p_{n}$ inside a face of the old embedding.

In the proof we will need the technical definitions from section 2, plus some additional ones given below.
Let $i, j, k$ be vertices belonging to the chains $C_{i}, C_{j}, C_{k}$ on the same face $F$ of a plane graph $G(P)$. The feasible region of the vertex $i$ is the set of points $p$ from which tangents at $p_{i}$ to the chain(s) $C_{i}$ can be drawn inside the face. The feasible region of a set of vertices $S=\{i, j\}$ or $S=\{i, j, k\}$ is the set of points $p$ for which all the line segments $p p_{s}, s \in S$ are tangents to the chains $C_{s}$ lying inside the face $F$. The pointed-feasible region of the triplet $\{i, j, k\}$ is the set of points $p$ such that the three line segments $p p_{i}, p p_{j}$ and $p p_{k}$ are tangents to the chains $C_{i}, C_{j}$ and $C_{k}$ (respectively), and meet at $p$ in a pointed fashion.

To obtain the feasible region of a vertex $i$, extend the two edges incident to the vertex to obtain a double wedge of lines emanating from $p_{i}$ and intersect them with the face. Figure 6 illustrates the feasibility region of a non-corner vertex inside a face of a pseudo-triangulation.

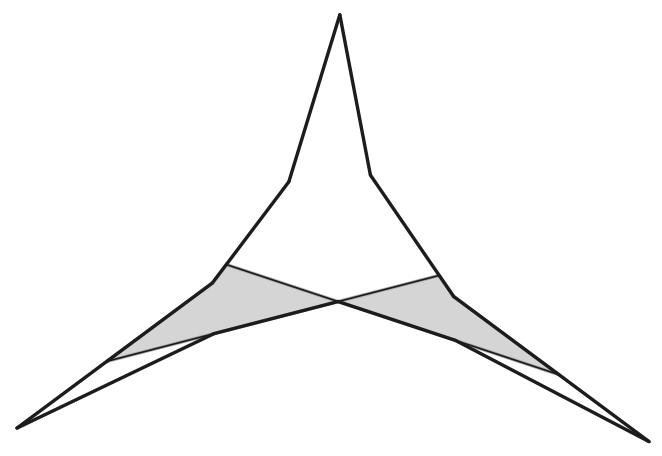

Figure 6: The feasible region for a non-corner vertex of a pseudo-triangle.

\section{Proof of Lemma 3.4 for a Henneberg I step.}

We have to show that it is always possible to find a position for the newly added vertex which maintains planarity and pointedness. If the current step is Henneberg I, the new vertex must be added inside one of the existing faces, and the edges must be tangent, at the other end, to the convex chains of the face. Since a vertex of degree 2 is always pointed, the newly added vertex is automatically so. Figure 7 illustrates the following simple observation: for any pair of points on the boundary of any interior face of a pseudotriangulation, their feasible region is non-empty and interior to the face. Placing the new vertex in this non-empty region completes the proof.

Proof of Lemma 3.4 for a Henneberg II step. In this case, an interior edge $i j$ is first removed, creating a face $F$ which is the union of the two faces incident to the edge before removal, and thus a pseudo-quadrilateral (possibly degenerate, if the removed edge was incident to a vertex of degree 2$)$. The new point $p_{n}$ must be connected to $p_{i}, p_{j}$ and a third vertex $p_{k}$ lying on the boundary of this face. To show that this is always possible, we have to prove that the pointed-feasible region of $\{i, j, k\}$ is non-empty. To structure the analysis into a small number of cases, we rely on the following simple observations about the feasible region of $\{i, j\}$.

- It consists of at most three polygonal regions (depending on which case from Figure 3 applies), connected in 


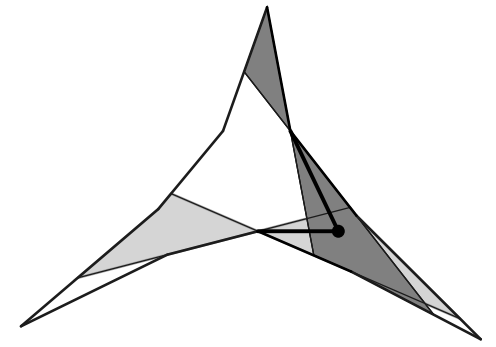

Figure 7: Feasible regions always intersect for a Henneberg I step.

a chain at the endpoints of the removed edge.

- The edge $p_{i}, p_{j}$ is interior to the middle polygonal region, which is a convex quadrilateral (a diamond shape).

- The extensions of the edge $p_{i}, p_{j}$ inside the face (if any of its endpoints is not a corner) are on the boundary of the other two polygonal regions.

Figure 8 illustrates all of the four possible shapes of the feasible region of a removed edge $\{i, j\}$.
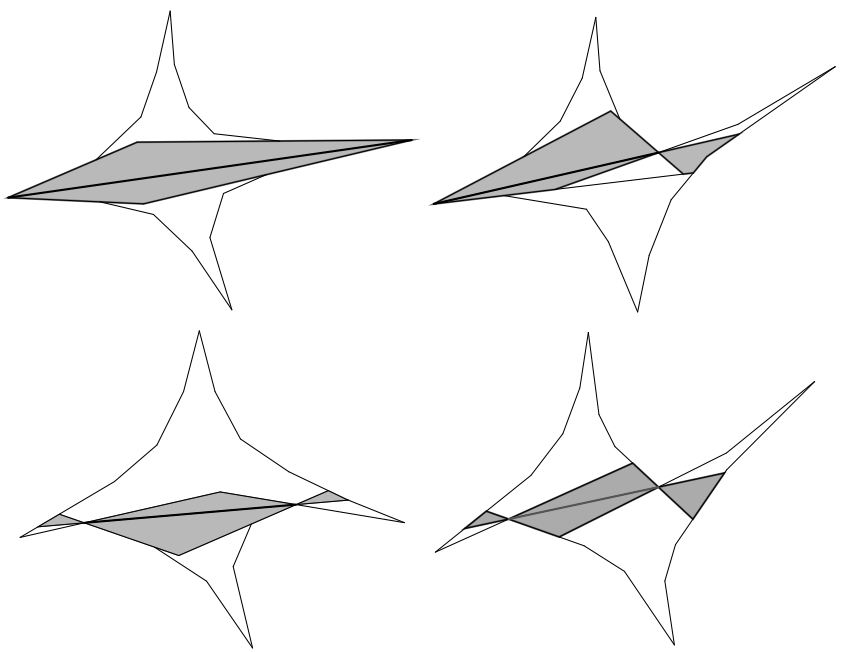

Figure 8: The feasible region of an interior removed edge: possible cases.

To complete the proof we must establish that the feasible region of the removed edge $\{i, j\}$ intersects the feasible region of any vertex $k$, and that the pointed-feasible region of $\{i, j, k\}$ is a non-empty subregion of this intersection.

Indeed, the feasible region of vertex $k$ intersects the supporting line of the edge $i j$ inside the face $F$ and hence cuts off an interval on it. Thus the intersection of the feasible region of vertex $k$ with that of the removed edge $\{i, j\}$ is a union of at most three polygons. By inspecting the four possibilities it is easy to see that a subregion of this intersection has the property that any point in it will be pointed, when joined by tangents to $p_{i}, p_{j}$ and $p_{k}$. The subcases are:
- The feasible region of $p_{k}$ intersects the middle (diamondshaped) region of $p_{i} p_{j}$. Then the pointed-feasible part of this region lies on the opposite side of the edge $p_{i} p_{j}$ than $p_{k}$.

- The feasible region of $p_{k}$ intersects one of the other (non-middle) regions. Then the pointed-feasible part of this region is the whole intersection.

Figure 9 illustrates the construction for the Henneberg II step when the feasible region of the removed edge has only one component, the diamond shaped region containing the removed edge.
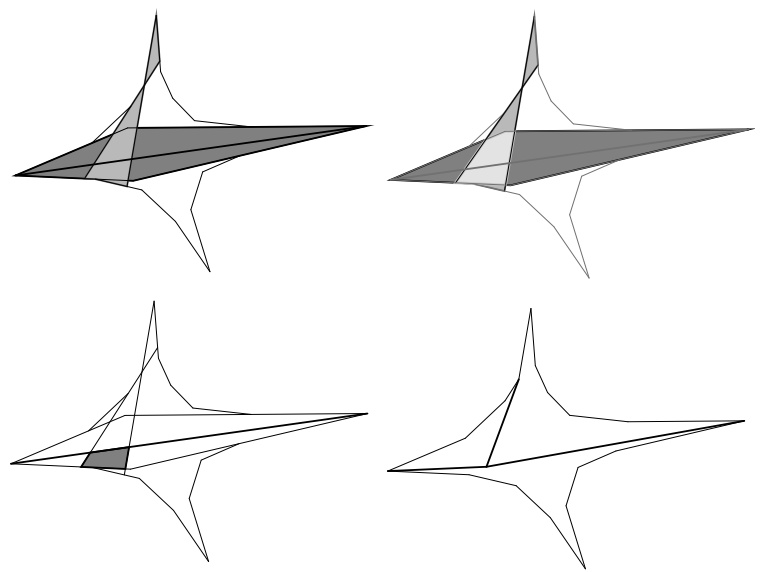

Figure 9: Henneberg II step on an interior face, where the feasible region of $p_{k}$ intersects the middle part of the feasible region of the removed edge: the two feasible regions, their intersection, the final pointed-feasible region and a placement of a pointed vertex and its three tangents.

Algorithmic analysis. The proof of the Main Theorem can be turned into an efficient time algorithm. Given a Laman graph, verifying its planarity and producing a topological embedding (stored as a quad-edge data structure [17] with face information) can be done in linear time [19]. One then chooses an outer face and in linear time one can perform the construction from Lemma 3.2 to get a triangular outer face. For producing a topological Henneberg construction, we keep an additional field in the vertex data structure, storing the degree of the vertex. We will keep two unordered lists containing the vertices of degree 2 and 3, respectively, To work out the Henneberg steps in reverse we need to do efficiently the following operations: a) select a vertex of minimum degree (which will be 2 or 3 ) and remove it, b) if the minimum degree is 3 , corresponding to a vertex $v$, we must find an edge $h$ that will be put back in after the removal of $v$, and c) restore the quad-edge data structure. Steps a) and c) can be done in constant time. Step b) requires deciding which of the three possibilities for $h$ among $v_{1} v_{2}, v_{1} v_{3}$ and $v_{2} v_{3}$ (where $v_{1}, v_{2}$ and $v_{3}$ are the neighbors of $v$ ) produces a Laman graph. Testing for the Laman condition on a graph with $2 n-3$ edges can be done by several algorithms in $O\left(n^{2}\right)$ : the algorithms of Imai [20] and Sugihara [40] via reductions to network flow or bipartite matching, or via ma- 
troid (tree) decompositions (see [48] and the references given there).

The algorithms of Imai [20] and Sugihara [40] use the following equivalent characterization of the Laman property.

Lemma 3.5. A graph $G$ with $n$ vertices and $m=2 n-3$ edges is a Laman graph if and only if, for every edge e, the multigraph " $G+3 e$ ", which is obtained by taking e four times, can be oriented in such a way that every vertex has indegree 2.

The condition on $G+3 e$ can be modeled as a flow problem on a bipartite network with "vertex nodes" and "edge nodes", where each vertex node has a supply of 2 and each edge node has a demand of 1, except for $e$ which has a demand of 4. (This is similar as in the proof of Lemma 4.1 below.) Since the total flow has value $O(n)$ and the network has size $O(n)$ such a flow can be found easily in $O\left(n^{2}\right)$ time by the algorithm of Ford and Fulkerson. A flow for the graph $G+3 e$ can be updated into a flow for graph $G+3 e^{\prime}$ for some other edge $e^{\prime}$ in $O(n)$ time, because this amounts to changing the demands by a total amount of only $O(1)$, and hence a new flow can be found by $O(1)$ flow augmentations, taking $O(n)$ time each.

When we apply a reverse Henneberg type II step and try to insert an edge $h$ into the reduced graph $G^{\prime}$, we only have to check the graph $\left(G^{\prime}+h\right)+3 h$ : Any subgraph which might violate the Laman condition for $G^{\prime}+h$ must clearly contain $h$, and then a feasible flow in $\left(G^{\prime}+h\right)+3 h$ would not exist. The total update of supplies and demands of the network from $G$ to $\left(G^{\prime}+h\right)+3 h$ still amounts to only $O(1)$, and hence we can perform a reverse Henneberg step in $O(n)$ time, which gives a total running time of $O\left(n^{2}\right)$ for the complete sequence of Henneberg reductions.

The embedding is done now by performing the Henneberg steps, starting with the outer triangular face embedded on an arbitrary initial triple of points. It is straightforward to see that each step takes constant time to determine a position for the new vertex, and the whole embedding takes linear time once the Henneberg sequence is known.

The time complexity would be improved by a positive answer to the following questions.

Open Question 2. Is it possible to decide the Laman condition in sub-quadratic (possibly linear) time for a planar graph?

Open Question 3. Is it possible to decide, faster than in linear time, which edge to put back in a Henneberg II step for a planar graph? For a combinatorial pseudo-triangulation?

\section{COMBINATORIAL PSEUDO-TRIANGULATIONS}

It follows from the Main Theorem that every planar embedding of a Laman graph has a combinatorial pseudo-triangulation assignment. But observe that in order to obtain this we do not need the full machinery and details of the proof of Lemma 3.4, only a combinatorial (and much simpler) version saying how to assign the convex and reflex angles at each inductive step, in a way consistent with the expectations of tangency for a real pseudo-triangulation.

In particular, this gives an efficient algorithmic way of computing a combinatorial pseudo-triangulation, or to enu- merate all of them, by iterating over all possible ways of performing a Henneberg step.

A different approach which gives an efficient algorithm right away is to reduce the problem of computing a combinatorial pseudo-triangulation to that of finding a maximum matching in a certain bipartite graph. This immediately gives an efficient algorithm.

Lemma 4.1. One can compute a combinatorial pseudotriangulation of a planar Laman graph in $O\left(n^{3 / 2}\right)$ time by solving a bipartite (multi-) matching problem.

Proof. (Sketch) Let $G=(V, E)$ be a planar Laman graph. We define a bipartite network $H$ with the two sets of the bipartition corresponding to the vertices and faces of $G$, and the edges representing incidences ("angles"). The pointed angles of a combinatorial pseudo-triangulation correspond to a subgraph of $H$ where each "vertex node" has degree 1 and each "face node" for $d_{i}$-sided face has degree $d_{i}-3$, with the exception of the exterior face which has degree $d_{i}$. A combinatorial pseudo-triangulation can thus be found in $O\left(n^{3 / 2}\right)$ time using the maximum flow algorithm of Dinits (see [41]), after finding a combinatorial planar embedding in linear time.

This set of degree-constrained subgraphs of a bipartite graph can be modelled as a network flow problem. Thus the set of combinatorial pseudo-triangulations is in one-to-one correspondence with the vertices of a polytope given by the equations and inequalities of the network flow.

The lemma allows us to find a combinatorial pseudo - triangulation because the existence of a combinatorial pseudotriangulation (even of a true pseudo-triangulation) has been established by other means in Theorem 1.1. However, the network flow model in the proof of Lemma 4.1 can also be adapted to a direct existence proof, using the Max-Flow/MinCut Theorem. The cut condition can be shown with the help of Euler's formula for planar graphs and the Laman condition.

A natural remaining question is whether it is always possible to embed a planar Laman graph when a combinatorial pseudo-triangulation assignment has been fixed a priori. The main result in this section is that this holds (and can be efficiently computed).

THEOREM 4.2. Every combinatorial pseudo-triangulation of a planar Laman graph can be realized with straight line segments.

The proof is based on ideas from the Tutte's theorem on convex embeddings [44], which was extended to directed graphs $[14,8]$. We draw the desired boundary face as a convex polygon. From a given combinatorial pseudo-triangulation $G$ we construct a directed graph $G^{*}$ having the same vertices as $G$ but not the same edges. At each interior vertex $v, G^{*}$ has three outgoing edges. Two of them are the extreme edges of $v$ in $G$ and the third is a new edge going through the interior of the pseudo-triangle containing the reflex angle at $v$, and joining $v$ to a vertex in a different chain of that pseudo-triangle (or to the opposite corner). We add these new edges in such a way that the resulting graph $G^{*}$ remains planarly embedded. To achieve this one can, for example, (topologically) triangulate each pseudo-triangle in such a way that only its three corners are ears of the triangulation, and use interior edges from this triangulation. 
The boundary edges of $G$ are considered as edges of $G^{*}$ too, but their orientations are not relevant (to be consistent with the outdegree- 3 constraint one can say they are oriented in both directions, because they are extreme edges of their two end-points).

Observe that some edges of $G^{*}$ may simultaneously get the two directions. For example, an edge of $G$ can be an extreme edge for its two end-points. The key result we will need about this graph $G^{*}$ is that it is 3 -connected in the following directed sense:

Lemma 4.3. Let $G^{*}$ be a directed graph obtained as above. Then, for any interior vertex $v$ and for any pair of forbidden vertices $u$ and $w$ there is a directed path in $G^{*}$ from $v$ to the boundary not passing through $u$ or $w$.

This means that if $G^{*}$ has a cutset consisting of two vertices, these two vertices must be on the boundary and $G^{*}$ is indeed 3-connected, if the boundary is a triangle. The proof of Theorem 4.2 then follows the same ideas of Tutte's proof that every 3-connected planar graph has an embedding with convex faces, see [32, pp. 122-132].

Time Analysis. In the proof of Theorem 4.2 (as in the original proof of Tutte's theorem for convex drawings of planar graphs), one writes a linear equation for each interior vertex, which says that the position of the vertex is the average of its (out-)neighbors. The position of the boundary vertices is fixed. It has been observed [7, Section 3.4] that the planar "structure" of this system of equations allows it to be solved in $O\left(n^{3 / 2}\right)$ time, using the $\sqrt{n}$-separator theorem for planar graphs in connection with the method of Generalized Nested Dissection (see $[24,25]$ or [33, Section 2.1.3.4]), or even in time $O(M(\sqrt{n}))$, where $M(n)=O\left(n^{2.375}\right)$ is the time to multiply two $n \times n$ matrices.

\section{PLANAR RIGIDITY CIRCUITS}

In this brief final section we sketch the extension of this result to rigidity circuits. A full version and applications are deferred to the full paper. The relevance of this extension is that it shows the possibility of having guaranteed linear embeddings for graphs that go beyond the minimal structure of Laman graphs. It is an open question how far into the dependent realm one can go, while guaranteeing straightline pseudo-triangular embeddings. The rigidity circuits are minimally dependent, in the rigidity matroid where the maximal independent sets are the Laman graphs. See [16] for the matroid-theoretic roots of this terminology.

A generic rigidity circuit is a simple graph with $2 n-2$ edges with the property that the removal of any edge produces a Laman graph. It has been shown recently [4] that 3-connected rigidity circuits admit a simple type of Henneberg construction using only Henneberg II.

A pseudo-triangulation circuit is a planar rigidity circuit embedded as a pseudo-triangulation. It is necessarily nonpointed, because pointed pseudo-triangulations are maximal pointed sets of non-crossing edges on any given planar point set [37]. See Figure 10 for an example of a rigidity circuit (a Hamiltonian polygon triangulation with an added edge between its two vertices of degree 2) and its embedding as a pseudo-triangulation with exactly one non-pointed vertex.

TheOREm 5.1. The analogue of the Main Result is true for embeddings of planar rigidity circuits: any planar generic
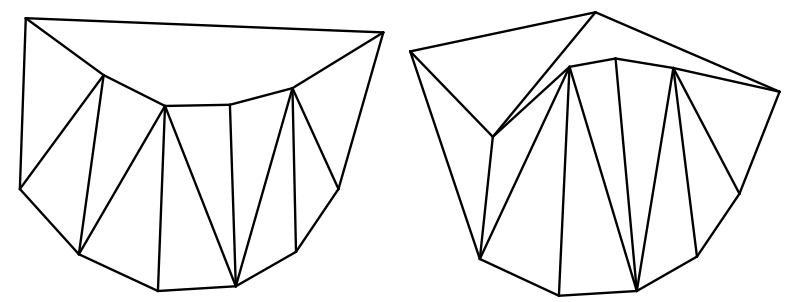

Figure 10: A rigidity circuit and one of its embeddings as a pseudo-triangulation with exactly one non-pointed vertex.

rigidity circuit can be embedded as a pseudo-triangulation with one non-pointed vertex.

Proof. (Sketch) We use the Berg-Jordán [4] inductive construction for 3-connected circuits and an inductive extension lemma similar to the one for Laman graphs. We then rely on Tutte's decomposition of 2-connected graphs into 3-connected components.

We may give an alternate proof by starting out with a combinatorial pseudo-triangulation for the rigidity circuit $C$ having one unmarked vertex $v$. Deleting an edge $e$ incident to $v$ and labeling, at $v$, the angle between the edges to the left and right of the deleted edge as $R$, induces a combinatorial pseudo-triangulation on $G=C-e$. The edge $e$ is added in the construction of $G^{*}$ and the resulting embedding of $C$ has the property that only vertex $v$ is nonpointed.

Acknowledgements. This research was initiated at the Workshop on Rigidity Theory and Scene Analysis organized by Ileana Streinu at the Bellairs Research Institute of McGill University in Barbados, Jan. 11-18, 2002 with partial support from NSF grant CCR-0203224.

\section{REFERENCES}

[1] O. Aichholzer, F. Aurenhammer, H. Krasser and B. Speckmann. Convexity minimizes pseudo-triangulations, Proc. 14th Canad. Conf. Comp. Geom., pp. 158-161, 2002.

[2] O. Aichholzer, B. Speckmann, G. Rote and I. Streinu. The zigzag path of a pseudo-triangulation, manuscript, 2003.

[3] P. Agarwal, J. Basch, L. J. Guibas, J. Herschberger and L. Zhang. Deformable free space tilings for kinetic collision detection, in Algorithmic and Computational Robotics: New Directions, Proc. 5th Workshop Algor. Found. Robotics (WAFR), 2001, pp. 83-96.

[4] A. Berg and T. Jordán. A proof of Connelly's conjecture on 3-connected circuits of the rigidity matroid. to appear in J. Combinatorial Theory, Ser. B, 2003.

[5] S. Bespamyatnikh. Enumerating pseudo-triangulations in the plane, in Proc. 14th Canad. Conf. Comp. Geom., 2002, pp. 162-166.

[6] H. Brönnimann, L. Kettner, M. Pocchiola and J. Snoeyink. Counting and enumerating pseudo-triangulations with the greedy flip algorithm, manuscript, 2001. 
[7] M. Chrobak, M. Goodrich and R. Tamassia. Convex drawings of graphs in two and three dimensions, In Proc. 12th Ann. Sympos. Comput. Geom., 1996, pp. 319-328.

[8] E. Colin de Verdière, M. Pocchiola and G. Vegter. Tutte's barycenter method applied to isotopies. In Proc. 13th Canad. Conf. Comput. Geom., August 2001. pp. 57-60. Extended version in ECG Report ECG-TR-124300-01, http://www-sop.inria.fr/ prisme/ECG/Results/Reports.html

[9] R. Connelly, E. Demaine and G. Rote. Straightening polygonal arcs and convexifying polygonal cycles, to appear, Discrete and Comp. Geometry, 2003. Preliminary version in Proc. 41st Symp. Found. of Comp. Science (FOCS), Redondo Beach, California, 2000, pp. 432-442.

[10] G. Di Battista, P. Eades, R. Tamassia and I. Tollis. Graph Drawing, Prentice Hall, 1999.

[11] T. Eren, W. Whiteley, A. Stephen Morse and P. Belhummeur. Sensor and Network Topologies of Formations with Distance-Direction Angle Constraints, submitted, March 2003.

[12] I. Fáry. On straight lines representation of planar graphs, Acta Sci. Math. Szeged 11 (1948), 229-233.

[13] S. Felsner. Convex drawings of planar graphs and the order dimension of 3-polytopes. Order 18 (2001), 19-37.

[14] M. S. Floater and C. Gotsman. How to morph tilings injectively, Journal of Computational and Applied Mathematics 101 (1999), 117-129.

[15] H. de Fraysseix, J. Pach and R. Pollack. How to draw a planar graph on a grid, Combinatorica 10 (1990), 41-51.

[16] J. Graver, B. Servatius and H. Servatius. Combinatorial Rigidity, Graduate Studies in Mathematics, vol. 2, Amer. Math. Soc., 1993.

[17] L. Guibas and J. Stolfi. Primitives for the manipulation of general subdivisions and the computation of Voronoi diagrams, ACM Trans. Graphics 4 (1985), 74-123.

[18] L. Henneberg. Die graphische Statik der starren Systeme, Leipzig 1911, Johnson Reprint 1968.

[19] J. E. Hopcroft and R. E. Tarjan, Efficient planarity testing, J. Assoc. Comput. Mach. 21 (1974), 549-568.

[20] H. Imai. On combinatorial structures of line drawings of polyhedra, Discrete Applied Mathematics, 10 (1985), 79-92.

[21] D. Jacobs, A. J. Rader, L. Kuhn, and M. Thorpe. Protein flexibility predictions using graph theory, Proteins 44 (2001), 150-165.

[22] L. Kettner, D. Kirkpatrick, A. Mantler, J. Snoeyink, B. Speckmann and F. Takeuchi. Tight degree bounds for pseudo-triangulations of points, Comp. Geom. Theory Applications, 2003, to appear.

[23] G. Laman. On graphs and rigidity of plane skeletal structures, J. Engineering Math. 4 (1970), 331-340.

[24] R. J. Lipton, D. J. Rose and R. E. Tarjan. Generalized nested dissection, SIAM J. Numer. Anal. 16 (1979), 346-358.

[25] R. J. Lipton and R. E. Tarjan. Applications of a planar separator theorem. SIAM J. Comput. 9, 1980,
615-627

[26] N. Mnëv. On manifolds of combinatorial types of projective configurations and convex polyhedra, Soviet Math. Doklady 32 (1985), 335-337.

[27] J. O'Rourke and I. Streinu. Vertex-edge pseudo-visibility graphs: characterization and recognition, in Proc. 13th ACM Symp. Comp. Geom, 1997, pp. 119-128.

[28] J. Pach and G. Tóth. Monotone drawings of planar graphs, in: Algorithms and Computation (P. Bose, P. Morin, eds.) LNCS 2518, Springer Verlag, 2002, pp. 647-653.

[29] M. Pocchiola and G. Vegter. Pseudo-triangulations: theory and applications, in Proc. 12th ACM Symp. Comput. Geometry, Philadelphia, 1996, pp. 291-300.

[30] M. Pocchiola and G. Vegter. Topologically sweeping visibility complexes via pseudo-triangulations, Discrete Comput. Geom. 16 (1996), 419-453.

[31] D. Randall, G. Rote, F. Santos and J. Snoeyink Counting triangulations and pseudo-triangulations of wheels, in Proc. 13th Canad. Conf. Comput. Geom., 2001, pp. 149-152.

[32] J. Richter-Gebert, Realization Spaces of Polytopes. Springer-Verlag, 1996.

[33] G. Rote Two solvable cases of the traveling salesman problem, Ph.D. Thesis, Technische Universität Graz, Institut für Mathematik, 1988.

[34] G. Rote, F. Santos and I. Streinu Expansive motions and the polytope of pointed pseudo-triangulations, in B. Aronov, S. Basu, J. Pach and M. Sharir (eds.), Discrete and Computational Geometry - The Goodman-Pollack Festschrift, Springer Verlag, to appear, 2003.

[35] B. Speckmann and C. Tóth. Allocating vertex $\pi$-guards in simple polygons via pseudo-triangulations, in Proc. 14th ACM-SIAM Symposium on Discrete Algorithms, 2003, pp. 109-118.

[36] I. Streinu. Stretchability of star-like pseudo-visibility graphs, in Proc. 15th ACM Symp. Comput. Geometry, 1999, pp. 274-280.

[37] I. Streinu. A combinatorial approach to planar non-colliding robot arm motion planning, in Proc. 41st Symp. Found. of Comp. Science (FOCS), Redondo Beach, California, 2000, pp. 443-453.

[38] I. Streinu. Combinatorial roadmaps in configuration spaces of simple planar polygons, to appear in S. Basu and L. Gonzalez-Vega (eds.), Proc. DIMACS Workshop on Algorithmic and Quantitative Aspects of Real Algebraic Geometry in Mathematics and Computer Science, 2003.

[39] W. Schnyder. Embedding planar graphs on the grid, in Proc. ACM-SIAM Symp. Discrete Algorithms (SODA), 1990, pp. 138-148.

[40] K. Sugihara. Detection of structural inconsistency in systems of equations with degrees of freedom and its applications, Discrete Applied Mathematics, 10 (1985), 297-312.

[41] R. E. Tarjan. Data Structures and Network Algorithms, CBMS-NSF Regional Conference Series in Applied Mathematics, Vol. 44, SIAM 1983.

[42] T. S. Tay and W. Whiteley. Generating isostatic 
graphs, Structural Topology 11 (1985), 21-68.

[43] M. F. Thorpe and P. M. Duxbury (eds.). Rigidity Theory and Applications, Kluwer Academic, 1999.

[44] W. T. Tutte, How to draw a graph, Proc. London Math. Soc., III Ser. 13 (1963) 743-768.

[45] W. T. Tutte, Convex representations of graphs, Proc. London Math. Soc., III Ser. 10 (1960), 304-320.

[46] W. Whiteley. Rigidity of molecular structures: generic and geometric analysis, in Rigidity Theory and Applications (M. F. Thorpe and P. M. Duxbury, eds.), Kluwer, 1999, pp. 21-46.

[47] W. Whiteley. Some matroids from discrete applied geometry, in J. E. Bonin et al. (ed.), Matroid theory, Contemporary Mathematics, vol. 197, Amer. Math. Soc., 1996, pp. 171-311,

[48] W. Whiteley. Rigidity and scene analysis, in Handbook of Discrete and Computational Geometry, (J. E. Goodman, J. O'Rourke, eds.), 1997, pp. 893-916. 